\title{
Wong-Zakai approximation of solutions to reflecting stochastic differential equations on domains in Euclidean spaces II
}

\author{
Shigeki Aida* \\ Mathematical Institute \\ Tohoku University, Sendai, 980-8578, JAPAN \\ e-mail: aida@math.tohoku.ac.jp
}

\begin{abstract}
The strong convergence of Wong-Zakai approximations of the solution to the reflecting stochastic differential equations was studied in [2]. We continue the study and prove the strong convergence under weaker assumptions on the domain.
\end{abstract}

\section{Introduction}

Wong-Zakai approximations of solutions of stochastic differential equations (=SDEs) were studied by many researchers, e.g. [15, 27, 13]. In the case of reflecting SDEs, Doss and Priouret [5] studied the Wong-Zakai approximations when the boundary is smooth. Actually, the unique existence of strong solutions of reflecting SDEs were proved for domains whose boundary may not be smooth by Tanaka [26], Lions-Sznitman [16] and Saisho [23]. In their studies, the standard conditions, (A), (B), (C) and admissibility condition, on the domain for reflecting SDEs were introduced and the unique existence of strong solutions were proved under the conditions either (A) and (B) hold or the domain is convex in [26] and [23]. We explain the conditions (A), (B), (C) in the next section. There were studies on Wong-Zakai approximations in such cases, e.g., [20, 21, 22] for convex domains and [7] for domains satisfying admissibility condition as well as conditions (A), (B), (C). When the domain is convex, Ren and $\mathrm{Xu}$ [22] proved that WongZakai approximations converge to the true solution in probability in the setting of stochastic variational inequality. In [2], the strong convergence of Wong-Zakai approximations was proved under the conditions (A), (B), (C). We note that Zhang [28] proved the strong convergence of Wong-Zakai approximations in the setting of [7] independent of [2]. The aim of this paper is to prove the strong convergence of Wong-Zakai approximation under the conditions either (A) and (B) hold or the domain is convex following the proof in [2]. Note that our proof in the case of convex domains is different from [22] and we give an estimate of the order of convergence.

The paper is organized as follows. In Section 2, we recall conditions of the boundary and state the main theorems. The first main theorem (Theorem 2.2) shows the strong convergence of Wong-Zakai approximations when the domain is convex. The estimate of the order of the convergence is the same as given in [2]. The second main theorem (Theorem 2.3) is concerned with the convergence of Wong-Zakai approximations in the case where the domain satisfies the conditions (A) and (B). We prove main theorems in Section 3 and Section 4.

${ }^{*}$ This research was partially supported by Grant-in-Aid for Scientific Research (B) No. 24340023. 


\section{Preliminaries and main theorems}

Let $D$ be a connected domain in $\mathbb{R}^{d}$. The following conditions can be found in [23]. In [2], we used the conditions (A), (B), (C) on $D$. In this paper, we will use (B') too. The set $\mathcal{N}_{x}$ of inward unit normal vectors at $x \in \partial D$ is defined by

$$
\begin{aligned}
\mathcal{N}_{x} & =\cup_{r>0} \mathcal{N}_{x, r}, \\
\mathcal{N}_{x, r} & =\left\{\boldsymbol{n} \in \mathbb{R}^{d}|| \boldsymbol{n} \mid=1, B(x-r \boldsymbol{n}, r) \cap D=\emptyset\right\}
\end{aligned}
$$

where $B(z, r)=\left\{y \in \mathbb{R}^{d}|| y-z \mid<r\right\}, z \in \mathbb{R}^{d}, r>0$.

Definition 2.1. (A) (uniform exterior sphere condition). There exists a constant $r_{0}>0$ such that

$$
\mathcal{N}_{x}=\mathcal{N}_{x, r_{0}} \neq \emptyset \quad \text { for any } x \in \partial D
$$

(B) There exist constants $\delta>0$ and $\beta \geq 1$ satisfying:

for any $x \in \partial D$ there exists a unit vector $l_{x}$ such that

$$
\left(l_{x}, \boldsymbol{n}\right) \geq \frac{1}{\beta} \quad \text { for any } \boldsymbol{n} \in \cup_{y \in B(x, \delta) \cap \partial D} \mathcal{N}_{y} .
$$

(B') (uniform interior cone condition) There exist $\delta>0$ and $0 \leq \alpha<1$ such that for any $x \in \partial D$ there exists a unit vector $l_{x}$ such that

$$
C\left(y, l_{x}, \alpha\right) \cap B(x, \delta) \subset \bar{D} \quad \text { for any } y \in B(x, \delta) \cap \partial D,
$$

where $C\left(y, l_{x}, \alpha\right)=\left\{z \in \mathbb{R}^{d}\left|\left(z-y, l_{x}\right) \geq \alpha\right| z-y \mid\right\}$.

(C) There exists a $C_{b}^{2}$ function $f$ on $\mathbb{R}^{d}$ and a positive constant $\gamma$ such that for any $x \in \partial D$, $y \in \bar{D}, \boldsymbol{n} \in \mathcal{N}_{x}$ it holds that

$$
(y-x, \boldsymbol{n})+\frac{1}{\gamma}((D f)(x), \boldsymbol{n})|y-x|^{2} \geq 0 .
$$

Note that if $D$ is a convex domain, the condition (A) holds for any $r_{0}$ and the condition (C) holds for $f \equiv 0$. Also we can prove that the condition (B') implies condition (B) with the same $\delta$ and $\beta=\left(1-\alpha^{2}\right)^{-1 / 2}$ by noting that $\boldsymbol{n}_{y} \in \mathcal{N}_{y, r}$ is equivalent to

$$
\left(z-y, \boldsymbol{n}_{y}\right)+\frac{1}{2 r}|y-z|^{2} \geq 0 \quad \text { for any } z \in \bar{D} .
$$

Further, if $D$ is a convex domain in $\mathbb{R}^{2}$ or a bounded convex domain in any dimensions, then the condition (B) holds. This is stated in [26]. Before considering reflecting SDE, let us explain the Skorohod problem on the multidimensional domain $D$ for which $\mathcal{N}_{x} \neq \emptyset$ for all $x \in \partial D$. Let $w=w(t)(0 \leq t \leq T)$ be a continuous path on $\mathbb{R}^{d}$ with $w(0) \in \bar{D}$. The pair of paths $(\xi, \phi)$ on $\mathbb{R}^{d}$ is a solution of a Skorohod problem associated with $w$ if the following properties hold.

(i) $\xi=\xi(t)(0 \leq t \leq T)$ is a continuous path in $\bar{D}$ with $\xi(0)=w(0)$. 
(ii) It holds that $\xi(t)=w(t)+\phi(t)$ for all $0 \leq t \leq T$.

(iii) $\phi=\phi(t)(0 \leq t \leq T)$ is a continuous bounded variation path on $\mathbb{R}^{d}$ such that $\phi(0)=0$ and

$$
\begin{aligned}
\phi(t) & =\int_{0}^{t} \mathbf{n}(s) d\|\phi\|_{[0, s]} \\
\|\phi\|_{[0, t]} & =\int_{0}^{t} 1_{\partial D}(\xi(s)) d\|\phi\|_{[0, s]} .
\end{aligned}
$$

where $\mathbf{n}(t) \in \mathcal{N}_{\xi(t)}$ if $\xi(t) \in \partial D$.

In the above, the notation $\|\phi\|_{[s, t]}$ stands for the total variation norm of $\phi(u)(0 \leq s \leq u \leq t \leq T)$.

Let us consider reflecting SDEs. Let $\sigma \in C_{b}^{2}\left(\mathbb{R}^{d} \rightarrow \mathbb{R}^{d} \otimes \mathbb{R}^{n}\right)$ and $b \in C_{b}^{1}\left(\mathbb{R}^{d} \rightarrow \mathbb{R}^{d}\right)$. Let $\Omega=C\left([0, \infty) \rightarrow \mathbb{R}^{n} ; \omega(0)=0\right)$ and $P$ be the Wiener measure on $\Omega$. Let $B(t, \omega)=\omega(t)(\omega \in \Omega)$ be the canonical realization of Brownian motion. We consider the reflecting SDE on $\bar{D}$ :

$$
X(t, x, \omega)=x+\int_{0}^{t} \sigma(X(s, x, \omega)) \circ d B(s, \omega)+\int_{0}^{t} b(X(s, x, \omega)) d s+\Phi(t, \omega),
$$

where $\circ d B(s)$ denotes the Stratonovich integral. We use the notation (SDE) $\sigma, b$ to indicate this equation. Note that this usage is different from that in [2] but I think there are no confusion. The solution $(X(t), \Phi(t))$ to this equation is nothing but a solution of the Skorohod problem associated with

$$
Y(t)=x+\int_{0}^{t} \sigma(X(s, x, \omega)) \circ d B(s, \omega)+\int_{0}^{t} b(X(s, x, \omega)) d s .
$$

As explained in the Introduction, if either the condition "(i) $D$ is a convex domain" or the condition "(ii) $D$ satisfies the conditions (A) and (B)" holds, then the strong solution $X(t)$ to (2.6) exists uniquely. These are due to Tanaka [26] for (i) and Saisho [23] for (ii). See also [16]. Let $X^{N}$ be the Wong-Zakai approximation of $X$. That is, $X^{N}$ is the solution to the reflecting differential equation driven by continuous bounded variation paths:

$$
X^{N}(t, x, \omega)=x+\int_{0}^{t} \sigma\left(X^{N}(s, x, \omega)\right) d B^{N}(s, \omega)+\int_{0}^{t} b\left(X^{N}(s, x, \omega)\right) d s+\Phi^{N}(t, \omega),
$$

where

$$
\begin{aligned}
& B^{N}(t)=B\left(t_{k-1}^{N}\right)+\frac{\Delta_{N} B_{k}}{\Delta_{N}}\left(t-t_{k-1}^{N}\right) \quad t_{k-1}^{N} \leq t \leq t_{k}^{N}, \\
& \Delta_{N} B_{k}=B\left(t_{k}^{N}\right)-B\left(t_{k-1}^{N}\right), \quad \Delta_{N}=\frac{T}{N}, \quad t_{k}^{N}=\frac{k T}{N} .
\end{aligned}
$$

We may denote $t_{k}^{N}$ and $\Delta_{N}$ by $t_{k}$ and $\Delta$ respectively. The solution $X^{N}$ uniquely exists under conditions (A) and (B) on D. See, e.g., 2, 23]. Under the convexity assumption of $D$ too, the solution $X^{N}$ uniquely exists by the results in [26]. In the convex case, we can check the existence in the following different way. More generally we consider a reflecting differential equation driven by a continuous bounded variation path $w_{t}$ :

$$
x_{t}=x_{0}+\int_{0}^{t} \sigma\left(x_{s}\right) d w_{s}+\int_{0}^{t} b\left(x_{s}\right) d s+\Phi(t) \quad x_{t} \in \bar{D} .
$$


The definition of the solution to this equation is similar to that of the equation previously discussed. Let $D_{R}=B\left(x_{0}, R\right) \cap D$. Then conditions (A) and (B) hold on $D_{R}$ and the solution, say, $x_{t}^{R}$ to reflecting differential equation on $D_{R}$ exists. Moreover by Lemma 2.4 in [2], $\left\|x^{R}\right\|_{[0, T]} \leq 2(\sqrt{2}+1)\left(\|\sigma\|_{\infty}\|w\|_{[0, T]}+\|b\|_{\infty} T\right)$, where $\|w\|_{[0, T]}$ denotes the total variation of $w(t) \quad(0 \leq t \leq T)$ as we already explained and $\|\sigma\|_{\infty}$ and $\|b\|_{\infty}$ denotes the supnorm of the operator norm and the Euclidean norm of $\sigma$ and $b$ respectively. Thus, we have $\max _{0 \leq t \leq T}\left|x^{R}(t)-x_{0}\right| \leq 2(\sqrt{2}+1)\left(\|\sigma\|_{\infty}\|w\|_{[0, T]}+\|b\|_{\infty} T\right)$ and we can apply the result in the case where (A) and (B) hold. Now we are in a position to state our main theorems.

Theorem 2.2. Assume $D$ is convex. Then, for any $0<\theta<1$, we have

$$
\begin{gathered}
\max _{0 \leq t \leq T} E\left[\left|X^{N}(t)-X(t)\right|^{2}\right] \leq C_{\theta} \cdot \Delta_{N}^{\theta / 2} \\
E\left[\max _{0 \leq t \leq T}\left|X^{N}(t)-X(t)\right|^{2}\right] \leq C_{T, \theta} \Delta_{N}^{\theta / 6} .
\end{gathered}
$$

Theorem 2.3. Assume the conditions (A) and (B) hold. Then for any $\varepsilon>0$, we have

$$
\lim _{N \rightarrow \infty} P\left(\max _{0 \leq t \leq T}\left|X^{N}(t)-X(t)\right| \geq \varepsilon\right)=0 .
$$

Remark 2.4. Rough path analysis clarifies the meaning of Wong-Zakai approximations. We refer the readers for basic results of rough path analysis to [17, 18, 19, 11, 12] and for WongZakai approximations of rough differential equations driven by fractional Brownian motions to [9, 14, 4. Note that reflecting differential equations driven by rough paths are defined and the existence and estimates of the solutions are studied in the author's recent paper [1]. See also [8] for reflecting differential equations driven by fractional Brownian motions whose Hurst parameter are greater than $1 / 2$.

\section{Convex domains}

In this section, we prove Theorem 2.2. Below, we use the notation

$$
\|w\|_{\infty,[s, t]}=\max _{s \leq u \leq v \leq t}|w(u)-w(v)| .
$$

The notation $\|w\|_{[s, t]}$ was already defined in Section 2. We can prove the following in the same way as in the proof of Lemma 2.3 in [2].

Lemma 3.1. Assume conditions (A) and (B) hold. Let $w$ be a q-variation continuous path such that

$$
|w(t)-w(s)| \leq \omega(s, t)^{1 / q} \quad 0 \leq s \leq t \leq T
$$

where $q \geq 1$ and $\omega$ is a control function. That $i s, \omega(s, t)$ is a nonnegative continuous function of $(s, t)$ with $0 \leq s \leq t \leq T$ satisfying $\omega(s, u)+\omega(u, t) \leq \omega(s, t)$ for all $0 \leq s \leq u \leq t \leq T$. Then the local time $\phi$ of the solution to the Skorohod problem associated with $w$ has the following estimate.

$$
\|\phi\|_{[s, t]} \leq \beta\left(\left\{\delta^{-1} G\left(\|w\|_{\infty,[s, t]}\right)+1\right\}^{q} \omega(s, t)+1\right)\left(G\left(\|w\|_{\infty,[s, t]}\right)+2\right)\|w\|_{\infty,[s, t]},
$$


where

$$
G(a)=4\left\{1+\beta \exp \left\{\beta(2 \delta+a) /\left(2 r_{0}\right)\right\}\right\} \exp \left\{\beta(2 \delta+a) /\left(2 r_{0}\right)\right\}
$$

The above estimate is one of key for the proof in [2]. Since the unbounded convex domains in $\mathbb{R}^{d}(d \geq 3)$ may not satisfy the condition (B), we cannot use this estimate. However, it is possible to estimate the total variation $\|\phi\|_{[s, t]}$ by $\|w\|_{\infty,[s, t]}$ together with the sup-norm of $\xi$ since we can give an estimate for the numbers $\beta$ and $\delta$ in the condition (B) for bounded convex domains.

Lemma 3.2. Let $D$ be a convex domain in $\mathbb{R}^{d}$. Let $x_{0} \in D$ and assume that there exists $R_{0}>0$ such that $\overline{B\left(R_{0}, x_{0}\right)} \subset D$. Let $R \geq R_{0}$ and define $D_{R}=D \cap B\left(R, x_{0}\right)$. The Condition (B) holds for the bounded convex domain $D_{R}$ with $\delta=R_{0} / 2$ and $\beta=\left(1+\left(\frac{2 R}{R_{0}}\right)^{2}\right)^{1 / 2}$.

Proof. We prove the condition (B'). Let $x \in \partial D_{R}$. Let $l_{x}$ be the unit vector in the direction from $x$ to $x_{0}$. Let $S\left(x_{0}\right)$ be a $d-1$ dimensional ball which is the slice of the ball $\overline{B\left(R_{0}, x_{0}\right)}$ by a hyperplane $H\left(x_{0}\right)$ that passes through $x_{0}$ and is orthogonal to $l_{x}$. Let $\alpha=\frac{R}{\sqrt{R^{2}+\left(R_{0} / 2\right)^{2}}}$. Then for any point $y \in B(\delta, x)$, it holds that $C\left(y, l_{x}, \alpha\right) \cap H\left(x_{0}\right) \subset S\left(x_{0}\right)$. Hence for any $y \in B(\delta, x) \cap \partial D_{R}, C\left(y, l_{x}, \alpha\right) \cap B(x, \delta) \subset \overline{D_{R}}$ which implies condition (B').

Lemma 3.3. Let $D$ be a convex domain. Let $x_{0} \in D$ and assume that there exists $R_{0}>0$ such that $\overline{B\left(R_{0}, x_{0}\right)} \subset D$. Let $w(t)(0 \leq t \leq T)$ be a continuous q-variation path with the control function $\omega$ on $\mathbb{R}^{d}$ with $w(0) \in \bar{D}$ and $q \geq 1$. Assume that there exists a solution $(\xi, \phi)$ to the Skorohod problem associated with $w$. Then it holds that

$$
\begin{aligned}
\|\phi\|_{[s, t]} \leq 10 & {\left[\left\{16 R_{0}^{-1}\left(1+4 R_{0}^{-2}\left\|\xi-x_{0}\right\|_{\infty,[0, T]}^{2}\right)^{1 / 2}+1\right\}^{q} \omega(s, t)+1\right] } \\
\times & \left(1+4 R_{0}^{-2}\left\|\xi-x_{0}\right\|_{\infty,[0, T]}^{2}\right)\|w\|_{\infty,[s, t]} .
\end{aligned}
$$

Proof. Note that $\xi$ is the solution of the Skorohod problem associated with $w$ on $\overline{D \cap B\left(x_{0}, R\right)}$, where $R=\left\|\xi-x_{0}\right\|_{\infty,[0, T]}$. This domain satisfies (B) with the constants $\delta$ and $\beta$ specified in the above lemma. In the lemma, letting $r_{0} \rightarrow \infty, G$ reads

$$
G(a)=4\left\{1+\sqrt{1+\left(2 R_{0}^{-1} R\right)^{2}}\right\} .
$$

By applying Lemma 3.1, we complete the proof.

To prove Theorem 2.2, we need moment estimates for increments of $X^{N}$ and $\Phi^{N}$.

Lemma 3.4. Assume $D$ is a convex domain. For the Wong-Zakai approximation $X^{N}$, we define

$$
Y^{N}(t, x, \omega)=x+\int_{0}^{t} \sigma\left(X^{N}(s, x, \omega)\right) d B^{N}(s, \omega)+\int_{0}^{t} b\left(X^{N}(s, x, \omega)\right) d s .
$$

(1) For all $p \geq 1$, we have 


$$
E\left[\left\|Y^{N}\right\|_{\infty,[s, t]}^{2 p}\right] \leq C_{p}|t-s|^{p}
$$

(2) Let $t_{k-1} \leq s<t \leq t_{k}$. Then we have for all $p \geq 1$,

$$
\begin{aligned}
& E\left[\left|X^{N}(t)-X^{N}(s)\right|^{2 p} \mid \mathcal{F}_{t_{k-1}}\right] \leq C_{p}|t-s|^{p}, \\
& \left\|\Phi^{N}\right\|_{[s, t]} \leq C\left(\left|\Delta B_{k}\right| \frac{t-s}{\Delta}+(t-s)\right)
\end{aligned}
$$

where $C_{p}$ and $C$ are positive constants.

Proof. These assertions can be proved by the same way as the proof of Lemma 4.3 and Lemma 4.4 in [2]. We assumed the condition (B) in those lemmas but we can argue in the same way since Skorohod equation associated with the continuous bounded variation path is uniquely solved under the convexity of $D$.

Lemma 3.5. Assume $D$ is convex. Let $p \geq 2$ be an integer. For $0 \leq s \leq t \leq T$, we have

$$
\begin{aligned}
E\left[|X(t)-X(s)|^{p}\right] & \leq C_{p}|t-s|^{p / 2}, \\
E\left[\left|X^{N}(t)-X^{N}(s)\right|^{p}\right] & \leq C_{p}|t-s|^{p / 2}, \\
E\left[\left\|\Phi^{N}\right\|_{[s, t]}^{p}\right] & \leq C_{p}|t-s|^{p / 2},
\end{aligned}
$$

where $C_{p}$ is a positive number independent of $N$.

Proof. Let $\tau_{R}=\inf \{t>0 \mid X(t, x, w) \notin B(x, R)\}$ and $X^{\tau_{R}}(t)=X\left(t \wedge \tau_{R}\right)$. For (3.10), it suffices to prove $E\left[\left|X^{\tau_{R}}(t)-X^{\tau_{R}}(s)\right|^{p} \mid\right] \leq C_{p}|t-s|^{p / 2}$ for all even positive integers $p$ and $0 \leq s \leq t \leq T$, where $C_{p}$ is independent of $R$. We prove this by an induction on $p$. Let $\tilde{b}=b+\frac{1}{2} \operatorname{tr}(D \sigma)(\sigma)$. By the Ito formula,

$$
\begin{aligned}
\left|X^{\tau_{R}}(t)-X^{\tau_{R}}(s)\right|^{2}= & 2 \int_{s \wedge \tau_{R}}^{t \wedge \tau_{R}}\left(X^{\tau_{R}}(u)-X^{\tau_{R}}(s), \sigma\left(X^{\tau_{R}}(u)\right) d B(u)\right) \\
& +2 \int_{s \wedge \tau_{R}}^{t \wedge \tau_{R}}\left(X^{\tau_{R}}(u)-X^{\tau_{R}}(s), \tilde{b}\left(X^{\tau_{R}}(u)\right)\right) d u \\
& +\int_{s \wedge \tau_{R}}^{t \wedge \tau_{R}} \operatorname{tr}\left(\left(\sigma^{t} \sigma\right)\left(X^{\tau_{R}}(u)\right)\right) d u \\
& +2 \int_{s \wedge \tau_{R}}^{t \wedge \tau_{R}}\left(X^{\tau_{R}}(u)-X^{\tau_{R}}(s), d \Phi(u)\right) .
\end{aligned}
$$

Noting the non-positivity of the term containing $\Phi$ which follows from the convexity of $D$ and taking the expectation, we have

$$
\begin{aligned}
& E\left[\left|X^{\tau_{R}}(t)-X^{\tau_{R}}(s)\right|^{2}\right] \\
& \leq C \int_{s}^{t} E\left[\left|X^{\tau_{R}}(u)-X^{\tau_{R}}(s)\right|^{2}\right] d u+C(t-s)
\end{aligned}
$$


which implies $E\left[\left|X^{\tau_{R}}(t)-X^{\tau_{R}}(s)\right|^{2}\right] \leq C(t-s)$. Let $p \geq 4$ and suppose the inequality holds for $p-2$.

$$
\begin{aligned}
\left|X^{\tau_{R}}(t)-X^{\tau_{R}}(s)\right|^{p} & \\
= & p \int_{s \wedge \tau_{R}}^{t \wedge \tau_{R}}\left|X^{\tau_{R}}(u)-X^{\tau_{R}}(s)\right|^{p-2}\left(X^{\tau_{R}}(u)-X^{\tau_{R}}(s), \sigma\left(X^{\tau_{R}}(u)\right) d B(u)\right) \\
& +p \int_{s \wedge \tau_{R}}^{t \wedge \tau_{R}}\left|X^{\tau_{R}}(u)-X^{\tau_{R}}(s)\right|^{p-2}\left(X^{\tau_{R}}(u)-X^{\tau_{R}}(s), \tilde{b}\left(X^{\tau_{R}}(u)\right)\right) d u \\
& +\frac{p}{2} \int_{s \wedge \tau_{R}}^{t \wedge \tau_{R}}\left|X^{\tau_{R}}(u)-X^{\tau_{R}}(s)\right|^{p-2} \operatorname{tr}\left(\left(\sigma^{t} \sigma\right)\left(X^{\tau_{R}}(u)\right)\right) d u \\
& +\frac{1}{2} p(p-2) \int_{s \wedge \tau_{R}}^{t \wedge \tau_{R}}\left|X^{\tau_{R}}(u)-X^{\tau_{R}}(s)\right|^{p-4} \mid{ }^{t} \sigma\left(\left.X^{\tau_{R}}(u)\left(X^{\tau_{R}}(u)-X^{\tau_{R}}(s)\right)\right|^{2} d u\right. \\
& +p \int_{s \wedge \tau_{R}}^{t \wedge \tau_{R}}\left|X^{\tau_{R}}(u)-X^{\tau_{R}}(s)\right|^{p-2}\left(X^{\tau_{R}}(u)-X^{\tau_{R}}(s), d \Phi(u)\right) .
\end{aligned}
$$

Hence we have

$$
\begin{aligned}
E\left[\left|X^{\tau_{R}}(t)-X^{\tau_{R}}(s)\right|^{p}\right] & \leq C_{p}\left(\int_{s}^{t} E\left[\left|X^{\tau_{R}}(t)-X^{\tau_{R}}(s)\right|^{p-2}\right]+E\left[\left|X^{\tau_{R}}(t)-X^{\tau_{R}}(s)\right|^{p-3}\right]\right) d u \\
& \leq C_{p}\left(\int_{s}^{t} E\left[\left|X^{\tau_{R}}(u)-X^{\tau_{R}}(s)\right|^{p-2}\right]+E\left[\left|X^{\tau_{R}}(u)-X^{\tau_{R}}(s)\right|^{p}\right]\right) d u
\end{aligned}
$$

which implies

$$
\begin{aligned}
E\left[\left|X^{\tau_{R}}(t)-X^{\tau_{R}}(s)\right|^{p}\right] & \leq C_{p} e^{C_{p}(t-s)} \int_{s}^{t} E\left[\left|X^{\tau_{R}}(u)-X^{\tau_{R}}(s)\right|^{p-2}\right] d u \\
& \leq C_{p}(t-s)^{p / 2} .
\end{aligned}
$$

This proves (3.10). Next we prove (3.11). Again, is is sufficient to prove the case where $p$ is an even number. We prove this by an induction on $p$ similarly to (3.10). By Lemma 2.4 in [2], we have $E\left[\left\|X^{N}\right\|_{[0, T]}^{p}\right]<\infty$ for any $p \geq 1$. We consider the case where $p=2$. Let $s=t_{l}<t_{m}=t$. By the chain rule,

$$
\begin{aligned}
\left|X^{N}(t)-X^{N}(s)\right|^{2} \\
=2 \int_{s}^{t}\left(X^{N}(u)-X^{N}(s), \sigma\left(X^{N}(u)\right) d B^{N}(u)\right)+2 \int_{s}^{t}\left(X^{N}(u)-X^{N}(s), b\left(X^{N}(u)\right)\right) d u \\
\quad+2 \int_{s}^{t}\left(X^{N}(u)-X^{N}(s), d \Phi^{N}(u)\right) \\
\leq 2 \int_{s}^{t}\left(X^{N}(u)-X^{N}(s), \sigma\left(X^{N}(u)\right) d B^{N}(u)\right)+2 \int_{s}^{t}\left(X^{N}(u)-X^{N}(s), b\left(X^{N}(u)\right)\right) d u \\
=: I_{1}+I_{2},
\end{aligned}
$$

where we have used the non-positivity of the third term which follows from the convexity of $D$. We estimate $I_{1}, I_{2}$. We have

$$
I_{1}=\sum_{k=l+1}^{m} 2 \int_{t_{k-1}}^{t_{k}}\left(X^{N}(u)-X^{N}(s), \sigma\left(X^{N}(u)\right) \frac{\Delta B_{k}}{\Delta}\right) d u .
$$




$$
\begin{aligned}
I_{1, k}:= & \int_{t_{k-1}}^{t_{k}}\left(X^{N}(u)-X^{N}(s), \sigma\left(X^{N}(u)\right) \frac{\Delta B_{k}}{\Delta}\right) d u \\
= & \left(X^{N}\left(t_{k-1}\right)-X^{N}(s), \sigma\left(X^{N}\left(t_{k-1}\right)\right) \Delta B_{k}\right) \\
& +\int_{t_{k-1}}^{t_{k}}\left(X^{N}(u)-X^{N}\left(t_{k-1}\right), \sigma\left(X^{N}\left(t_{k-1}\right)\right) \frac{\Delta B_{k}}{\Delta}\right) d u \\
& +\int_{t_{k-1}}^{t_{k}}\left(X^{N}\left(t_{k-1}\right)-X^{N}(s),\left(\sigma\left(X^{N}(u)\right)-\sigma\left(X^{N}\left(t_{k-1}\right)\right)\right) \frac{\Delta B_{k}}{\Delta}\right) \\
& +\int_{t_{k-1}}^{t_{k}}\left(X^{N}(u)-X^{N}\left(t_{k-1}\right),\left(\sigma\left(X^{N}(u)\right)-\sigma\left(X^{N}\left(t_{k-1}\right)\right)\right) \frac{\Delta B_{k}}{\Delta}\right) d u
\end{aligned}
$$

By Lemma 3.4 (2),

$$
\begin{aligned}
E\left[I_{1, k}\right] & \leq C\left(1+E\left[\left|X^{N}\left(t_{k-1}\right)-X^{N}(s)\right|\right]\right) \Delta \\
& \leq C\left(\int_{t_{k-1}}^{t_{k}}\left(E\left[\left|X^{N}(u)-X^{N}(s)\right|^{2}\right]+1\right) d u\right) .
\end{aligned}
$$

Thus, we obtain

$$
E\left[\left|X^{N}(t)-X^{N}(s)\right|^{2}\right] \leq C\left((t-s)+\int_{s}^{t} E\left[\left|X^{N}(u)-X^{N}(s)\right|^{2}\right] d u\right) .
$$

Again by noting Lemma 3.4 (2), we see that (3.20) holds for any $0 \leq s \leq t \leq T$. Applying the Gronwall inequality, we get the inequality (3.11) with $p=2$. Let $p \geq 4$. Let $s=t_{l}<t_{m}=t$. By the chain rule,

$$
\begin{aligned}
\left|X^{N}(t)-X^{N}(s)\right|^{p} & =p \int_{s}^{t}\left|X^{N}(u)-X^{N}(s)\right|^{p-2}\left(X^{N}(u)-X^{N}(s), \sigma\left(X^{N}(u)\right) d B^{N}(u)\right) \\
& +p \int_{s}^{t}\left|X^{N}(u)-X^{N}(s)\right|^{p-2}\left(X^{N}(u)-X^{N}(s), b\left(X^{N}(u)\right)\right) d u \\
& +p \int_{s}^{t}\left|X^{N}(u)-X^{N}(s)\right|^{p-2}\left(X^{N}(u)-X^{N}(s), d \Phi^{N}(u)\right) \\
& \leq p \int_{s}^{t}\left|X^{N}(u)-X^{N}(s)\right|^{p-2}\left(X^{N}(u)-X^{N}(s), \sigma\left(X^{N}(u)\right) d B^{N}(u)\right) \\
& +p \int_{s}^{t}\left|X^{N}(u)-X^{N}(s)\right|^{p-2}\left(X^{N}(u)-X^{N}(s), b\left(X^{N}(u)\right)\right) d u \\
& =: J_{1}+J_{2},
\end{aligned}
$$

where we have used the non-positivity of the third term which follows from the convexity of $D$. By noting $\left|X^{N}(u)-X^{N}(s)\right|^{p-1} \leq \frac{1}{2}\left(\left|X^{N}(u)-X^{N}(s)\right|^{p}+\left|X^{N}(u)-X^{N}(s)\right|^{p-2}\right)$ and by the assumption of induction, we have

$$
E\left[J_{2}\right] \leq C(t-s)^{p / 2}+\int_{s}^{t} E\left[\left|X^{N}(u)-X^{N}(s)\right|^{p}\right] d u .
$$


For $J_{1}$, we have

$$
\begin{aligned}
& J_{1}=\sum_{k=l+1}^{m} p \int_{t_{k-1}}^{t_{k}}\left|X^{N}(u)-X^{N}(s)\right|^{p-2}\left(X^{N}(u)-X^{N}(s), \sigma\left(X^{N}(u)\right) \frac{\Delta B_{k}}{\Delta}\right) d u . \\
& \int_{t_{k-1}}^{t_{k}}\left|X^{N}(u)-X^{N}(s)\right|^{p-2}\left(X^{N}(u)-X^{N}(s), \sigma\left(X^{N}(u)\right) \frac{\Delta B_{k}}{\Delta}\right) d u \\
& =\int_{t_{k-1}}^{t_{k}}\left|X^{N}(u)-X^{N}(s)\right|^{p-2}\left(X^{N}\left(t_{k-1}\right)-X^{N}(s), \sigma\left(X^{N}\left(t_{k-1}\right)\right) \frac{\Delta B_{k}}{\Delta}\right) d u \\
& +\int_{t_{k-1}}^{t_{k}}\left|X^{N}(u)-X^{N}(s)\right|^{p-2}\left(X^{N}(u)-X^{N}\left(t_{k-1}\right), \sigma\left(X^{N}\left(t_{k-1}\right)\right) \frac{\Delta B_{k}}{\Delta}\right) d u \\
& \quad+\int_{t_{k-1}}^{t_{k}}\left|X^{N}(u)-X^{N}(s)\right|^{p-2}\left(X^{N}\left(t_{k-1}\right)-X^{N}(s),\left(\sigma\left(X^{N}(u)\right)-\sigma\left(X^{N}\left(t_{k-1}\right)\right)\right) \frac{\Delta B_{k}}{\Delta}\right) \\
& +\int_{t_{k-1}}^{t_{k}}\left|X^{N}(u)-X^{N}(s)\right|^{p-2}\left(X^{N}(u)-X^{N}\left(t_{k-1}\right),\left(\sigma\left(X^{N}(u)\right)-\sigma\left(X^{N}\left(t_{k-1}\right)\right)\right) \frac{\Delta B_{k}}{\Delta}\right) d u \\
& =J_{1,1}^{k}+J_{1,2}^{k}+J_{1,3}^{k}+J_{1,4}^{k} .
\end{aligned}
$$

We have

$$
J_{1,1}^{k}=J_{1,1,1}^{k}+J_{1,1,2}^{k}+J_{1,1,3}^{k}
$$

where

$$
\begin{aligned}
J_{1,1,1}^{k}=\int_{t_{k-1}}^{t_{k}} & \left\{\int_{t_{k-1}}^{u}(p-2)\left|X^{N}(r)-X^{N}(s)\right|^{p-4}\left(X^{N}(r)-X^{N}(s), \sigma\left(X^{N}(r)\right) \frac{\Delta B_{k}}{\Delta}\right) d r\right\} \\
\times & \left(X^{N}\left(t_{k-1}\right)-X^{N}(s), \sigma\left(X^{N}\left(t_{k-1}\right)\right) \frac{\Delta B_{k}}{\Delta}\right) d u \\
J_{1,1,2}^{k}= & \int_{t_{k-1}}^{t_{k}}\left\{\int_{t_{k-1}}^{u}(p-2)\left|X^{N}(r)-X^{N}(s)\right|^{p-4}\left(X^{N}(r)-X^{N}(s), b\left(X^{N}(r)\right)\right) d r\right\} \\
& \times\left(X^{N}\left(t_{k-1}\right)-X^{N}(s), \sigma\left(X^{N}\left(t_{k-1}\right)\right) \frac{\Delta B_{k}}{\Delta}\right) d u \\
J_{1,1,3}^{k}= & \int_{t_{k-1}}^{t_{k}}\left\{\int_{t_{k-1}}^{u}(p-2)\left|X^{N}(r)-X^{N}(s)\right|^{p-4}\left(X^{N}(r)-X^{N}(s), d \Phi^{N}(r)\right)\right\} \\
& \times\left(X^{N}\left(t_{k-1}\right)-X^{N}(s), \sigma\left(X^{N}\left(t_{k-1}\right)\right) \frac{\Delta B_{k}}{\Delta}\right) d u
\end{aligned}
$$

By the estimate for $p=2$ and Lemma 3.4 (2), we have

$$
\begin{aligned}
E\left[J_{1,1,1}^{k}\right] & \leq C_{p} E\left[\left|X^{N}\left(t_{k-1}\right)-X^{N}(s)\right|^{p-2}\right] \Delta \\
& +\int_{t_{k-1}}^{t_{k}} \int_{t_{k-1}}^{u} E\left[\left|X^{N}(r)-X^{N}\left(t_{k-1}\right)\right|^{p-3}|| X^{N}\left(t_{k-1}\right)-X^{N}(s) \mid\left(\frac{\left|\Delta B_{k}\right|}{\Delta}\right)^{2}\right] d r d u \\
& \leq C_{p} E\left[\left|X^{N}\left(t_{k-1}\right)-X^{N}(s)\right|^{p-2}\right] \Delta+C\left(t_{k-1}-s\right)^{1 / 2} \Delta^{(p-1) / 2}
\end{aligned}
$$


Noting that for any $a>0, \sum_{k=l+1}^{m}\left(t_{k-1}-s\right)^{a} \Delta \leq \int_{s}^{t}(u-s)^{a} d u \leq(t-s)^{a+1} /(a+1)$ and using the assumption of induction,

$$
E\left[\sum_{k=l+1}^{m} J_{1,1,1}^{k}\right] \leq C \sum_{k=l+1}^{m}\left\{\left(t_{k-1}-s\right)^{(p-2) / 2} \Delta+\left(t_{k-1}-s\right)^{1 / 2} \Delta^{(p-1) / 2}\right\} \leq C(t-s)^{p / 2} .
$$

Similarly,

$$
\begin{gathered}
E\left[J_{1,1,2}^{k}\right] \leq C_{p} E\left[\left|X^{N}\left(t_{k-1}\right)-X^{N}(s)\right|^{p-2}\right] \Delta^{3 / 2}+C\left(t_{k-1}-s\right)^{1 / 2} \Delta^{p / 2} . \\
E\left[J_{1,1,3}^{k}\right] \leq C_{p} E\left[\left|X^{N}\left(t_{k-1}\right)-X^{N}(s)\right|^{p-2} E\left[\left\|\Phi^{N}\right\|_{\left[t_{k-1}, t_{k}\right]}\left|\Delta B_{k}\right| \mid \mathcal{F}_{t_{k-1}}\right]\right] \\
+C_{p} E\left[\left|X^{N}\left(t_{k-1}\right)-X^{N}(s)\right| E\left[\max _{t_{k-1} \leq r \leq t_{k}}\left|X^{N}(r)-X^{N}\left(t_{k-1}\right)\right|^{p-3}\left\|\Phi^{N}\right\|_{t_{k-1}, t_{k}}\left|\Delta B_{k}\right| \mid \mathcal{F}_{t_{k-1}}\right]\right] \\
\leq C_{p} E\left[\left|X^{N}\left(t_{k-1}\right)-X^{N}(s)\right|\right]^{p-2} \Delta+\left(t_{k-1}-s\right)^{1 / 2} \Delta^{(p-1) / 2} .
\end{gathered}
$$

Thus, we have $E\left[\sum_{k=l+1}^{m} J_{1,1,2}^{k}\right]+E\left[\sum_{k=l+1}^{m} J_{1,1,3}^{k}\right] \leq C(t-s)^{p / 2}$.

We consider the terms $J_{1, i}^{k}(2 \leq i \leq 4)$.

$$
\begin{aligned}
E\left[J_{1,2}^{k}\right] \leq & \Delta^{-1} \int_{t_{k-1}}^{t_{k}} E\left[\left|X^{N}\left(t_{k-1}\right)-X^{N}(s)\right|^{p-2} E\left[\left|X^{N}(u)-X^{N}\left(t_{k-1}\right)\right|^{p-1}\left|\Delta B_{k}\right| \mid \mathcal{F}_{t_{k-1}}\right]\right] d u \\
& +\Delta^{p / 2} \leq \\
\leq & E\left[\left|X^{N}\left(t_{k-1}\right)-X^{N}(s)\right|^{p-2}\right] \Delta^{p / 2}+\Delta^{p / 2} . \\
E\left[J_{1,3}^{k}\right] \leq & C \Delta^{-1} \int_{t_{k-1}}^{t_{k}} E\left[\left|X^{N}\left(t_{k-1}\right)-X^{N}(s)\right| E\left[\left|X^{N}(u)-X^{N}\left(t_{k-1}\right)\right|^{p-1}\left|\Delta B_{k}\right| \mid \mathcal{F}_{t_{k-1}}\right]\right] d u \\
& +C \Delta^{-1} \int_{t_{k-1}}^{t_{k}} E\left[\left|X^{N}\left(t_{k-1}\right)-X^{N}(s)\right|^{p-1} E\left[\left|X^{N}(u)-X^{N}\left(t_{k-1}\right)\right|\left|\Delta B_{k}\right| \mid \mathcal{F}_{t_{k-1}}\right]\right] d u \\
\leq & C E\left[\left|X^{N}\left(t_{k-1}\right)-X^{N}(s)\right|\right] \Delta^{p / 2}+C E\left[\left|X^{N}\left(t_{k-1}\right)-X^{N}(s)\right|^{p-1}\right] \Delta . \\
E\left[J_{1,4}^{k}\right] \leq & C \Delta^{-1} \int_{t_{k-1}}^{t_{k}} E\left[\left|X^{N}\left(t_{k-1}\right)-X^{N}(s)\right|^{p-2} E\left[\left|X^{N}(u)-X^{N}\left(t_{k-1}\right)\right|^{2}\left|\Delta B_{k}\right| \mid \mathcal{F}_{t_{k-1}}\right]\right] d u \\
& +C \Delta^{-1} \int_{t_{k-1}}^{t_{k}} E\left[\left|X^{N}(u)-X^{N}\left(t_{k-1}\right)\right|^{p}\left|\Delta B_{k}\right|\right] d u \\
\leq & C E\left[\left|X^{N}\left(t_{k-1}\right)-X^{N}(s)\right|^{p-2}\right] \Delta^{3 / 2}+C \Delta^{(p+1) / 2} .
\end{aligned}
$$

Hence

$$
E\left[\left|X^{N}(t)-X^{N}(s)\right|^{p}\right] \leq C(t-s)^{p / 2}+\int_{s}^{t} E\left[\left|X^{N}(u)-X^{N}(s)\right|^{p}\right] d u .
$$

By using (3.8), we see that (3.36) holds for any $0 \leq s \leq t \leq T$. By the Gronwall inequality, we get the desired inequality for $p$ and we complete the proof of (3.11). The estimate (3.7) and the Garsia-Rodemich-Rumsey estimate imply the $L^{r}$-boundedness of the Hölder norm with exponent $1 / 2-\varepsilon$ of $Y^{N}$ for any $r \geq 1$ and $0<\varepsilon<1 / 2$. Hence, (3.12) follows from Lemma 3.3 and (3.11). 
Thanks to the above estimates, we can prove the first main theorem as in [2].

Proof of Theorem 2.2. Let $X_{E}^{N}(t)$ be the Euler approximation of $X$. That is, $X_{E}^{N}(0)=x$ and $X_{E}^{N}$ is the solution to the Skorohod equation:

$$
\begin{aligned}
X_{E}^{N}(t)= & X_{E}^{N}\left(t_{k-1}^{N}\right)+\sigma\left(X_{E}^{N}\left(t_{k-1}^{N}\right)\right)\left(B(t)-B\left(t_{k-1}^{N}\right)\right)+\tilde{b}\left(X_{E}^{N}\left(t_{k-1}^{N}\right)\right)\left(t-t_{k-1}^{N}\right) \\
& +\Phi_{E}^{N}(t)-\Phi^{N}\left(t_{k-1}^{N}\right) \quad t_{k-1}^{N} \leq t \leq t_{k}^{N},
\end{aligned}
$$

where $\Phi_{E}^{N}(t)-\Phi^{N}\left(t_{k-1}^{N}\right)$ is the local time term and $\tilde{b}=b+\frac{1}{2} \operatorname{tr}(D \sigma)(\sigma)$. By a similar argument to (3.10) and (3.12), we obtain

$$
\begin{gathered}
E\left[\left\|X_{E}^{N}\right\|_{\infty,[s, t]}^{2 p}\right] \leq C_{p}|t-s|^{p}, \\
E\left[\left\|\Phi_{E}^{N}\right\|_{[s, t]}^{2 p}\right] \leq C_{p}|t-s|^{p} .
\end{gathered}
$$

Hence by the same proof as in [2], we obtain there exists $C_{p}>0$ such that

$$
E\left[\max _{0 \leq t \leq T}\left|X_{E}^{N}(t)-X(t)\right|^{2 p}\right] \leq C_{p} \Delta_{N}^{p}
$$

By these estimates and Lemma 3.5. we can prove the desired estimates as in the same way in [2]. The proof is simpler than that in [2] because $f \equiv 0$ when $D$ is convex.

\section{General domains satisfying conditions (A) and (B)}

In this section, we prove Theorem 2.3. The following observation which can be found in Lemma 5.3 in 23$]$ is crucial for our purpose.

Lemma 4.1. Assume (A) and (B) are satisfied on D. Let $\gamma=2 r_{0} \beta^{-1}$. Then for for each $z_{0} \in \partial D$ we can find a function $f \in C_{b}^{2}\left(\mathbb{R}^{d}\right)$ satisfying (2.3) for any $x \in B\left(z_{0}, \delta\right) \cap \partial D, y \in \bar{D}$ and $\boldsymbol{n} \in \mathcal{N}_{x}$. Moreover the sup-norms $\left\|D^{k} f\right\|_{\infty}(k=0,1,2)$ are bounded by some constant independent of $z_{0}$.

It is stated in Lemma 5.3 in [23] that the conclusion in the above proposition holds for $y \in B\left(z_{0}, \delta\right) \cap \bar{D}$. However, it is obvious to see the same conclusion holds for any $y \in \bar{D}$. Thanks to this proposition, we can localize the problem. Let us choose a positive number $\delta^{\prime}<\delta / 2$. For any $z \in \bar{D}$, if $B\left(z, \delta^{\prime}\right) \cap \partial D \neq \emptyset$, then there exists $z_{0} \in \partial D$ such that $\overline{B\left(z, \delta^{\prime}\right)} \subset B\left(z_{0}, \delta\right)$. Next, let $\chi$ be a $C^{\infty}$ function on $\mathbb{R}^{d}$ such that $\chi(x)=1$ for $x$ with $|x| \leq \delta^{\prime} / 2, \chi(x)=0$ for $x$ with $|x| \geq 2 \delta^{\prime} / 3$. Let $z \in \bar{D}$ and define

$$
\sigma^{z}(x)=\sigma(x) \chi(x-z), \quad b^{z}(x)=b(x) \chi(x-z) \quad x \in \mathbb{R}^{d} .
$$

We denote the solution and the Wong-Zakai approximation to $(\mathrm{SDE})_{\sigma^{z}, b^{z}}$ with the starting point $x$ by $X^{z}(t, x, \omega)$ and $X^{N, z}(t, x, \omega)$ respectively. By the uniqueness of strong solutions, we have

(i) $X^{z}(t, x, \omega)=X^{N, z}(t, x, \omega)=x$ for all $x \in B\left(z, \delta^{\prime} / 2\right)^{c}$

(ii) If $x \in B\left(z, 2 \delta^{\prime} / 3\right)$, then both $X^{z}(t, x, \omega)$ and $X^{N, z}(t, x, \omega)$ belong to $B\left(z, 2 \delta^{\prime} / 3\right)$ for all $t$ and $N$. 
We need a continuous dependence of solutions of reflecting SDE with respect to the starting point as in the following. Below, we state it for the particular case $\mathrm{SDE}_{\sigma^{z}, b^{z}}$ but it is easy to extend the result to more general situations.

Lemma 4.2. Assume (A) and (B) hold on D.

(1) For any $p \geq 1$ and $x, y \in \bar{D}$, we have

$$
E\left[\max _{0 \leq t \leq T}\left|X^{z}(t, x)-X^{z}(t, y)\right|^{p}\right] \leq C_{p}|x-y|^{p} .
$$

The constant $C_{p}$ is independent of $z$.

(2) Let $0<\theta<1$. There exists a positive constant $C_{T, \theta}$ such that for any $x, z \in \bar{D}$, we have

$$
E\left[\max _{0 \leq t \leq T}\left|X^{N, z}(t, x)-X^{z}(t, x)\right|^{2}\right] \leq C_{T, \theta} \Delta_{N}^{\theta / 6}
$$

(3) Let $x \in B\left(z, \delta^{\prime} / 2\right)$. Let $\tau(\omega)$ and $\sigma(\omega)$ be the exit time of $X(t, x, \omega)$ and $X^{z}(t, x, \omega)$ respectively from $B\left(z, \delta^{\prime} / 2\right)$. Then $\tau(\omega)=\sigma(\omega)$ P-a.s. $\omega$ and $X(t, x, \omega)=X^{z}(t, x, \omega)(0 \leq$ $t \leq \tau(\omega))$.

Proof. (1) If $x$ or $y$ belongs to $B\left(z, 2 \delta^{\prime} / 3\right)^{c}$, the assertion is true because of (i) and (ii) above. Therefore we may assume $x, y \in B\left(z, 2 \delta^{\prime} / 3\right)$. Suppose $B\left(z, \delta^{\prime}\right) \cap \partial D$ is not an empty set. Then we can pick a point $z_{0} \in B\left(z, \delta^{\prime}\right) \cap \partial D$ such that $B\left(z, \delta^{\prime}\right) \subset B\left(z_{0}, \delta\right)$. Let $f$ be a function in Lemma 4.1 associated with $z_{0}$. Let

$$
Z^{z}(t)=X^{z}(t, x)-X^{z}(t, y), \quad \rho^{z}(t)=e^{-\frac{2}{\gamma}\left(f\left(X^{z}(t, x)\right)+f\left(X^{z}(t, y)\right)\right.}, \quad k^{z}(t)=\rho^{z}(t)\left|Z^{z}(t)\right|^{2} .
$$

In the calculation below, we omit the superscript $z$ in the notation $X^{z}$, and so on. Let $\tilde{b}=$ $b+\frac{1}{2} \operatorname{tr}(D \sigma)(\sigma)$. By the Ito formula,

$$
\begin{aligned}
d k(t) & \\
= & \rho(t)\{2(Z(t),(\sigma(X(t, x))-\sigma(X(t, y))) d B(t)) \\
& \left.+2(Z(t), \tilde{b}(X(t, x))-\tilde{b}(X(t, y))) d t+\|\sigma(X(t, x))-\sigma(X(t, y))\|_{H . S .}^{2} d t\right\} \\
& +2 \rho(t)(Z(t), d \Phi(t, x)-d \Phi(t, y)) \\
& -\frac{2 \rho(t)}{\gamma}|Z(t)|^{2}\{((D f)(X(t, x)), d \Phi(t, x))+((D f)(X(t, y)), d \Phi(t, y))\} \\
& -\frac{2 \rho(t)}{\gamma}|Z(t)|^{2}\{((D f)(X(t)), \sigma(X(t, x)) d B(t))+((D f)(X(t, y)), \sigma(X(t, y)) d B(t))\} \\
& +R(t) d t,
\end{aligned}
$$


where

$$
\begin{aligned}
R(t)= & \frac{4 \rho(t)}{\gamma}\left((D f)(X(t, x)), \sigma(X(t, x))^{t}(\sigma(X(t, x))-\sigma(X(t, y)))(Z(t))\right) d t \\
& +\frac{4 \rho(t)}{\gamma}\left((D f)(X(t, y)), \sigma(X(t, y))^{t}(\sigma(X(t, x))-\sigma(X(t, y)))(Z(t))\right) d t \\
& -\frac{2 \rho(t)}{\gamma}|Z(t)|^{2}(((D f)(X(t, x)), \tilde{b}(X(t, x))) d t+((D f)(X(t, y)), \tilde{b}(X(t, y))) d t) \\
& -\frac{\rho(t)}{\gamma}|Z(t)|^{2}\left\{\operatorname{tr}\left(D^{2} f\right)(X(t, x))(\sigma(X(t, x)) \cdot, \sigma(X(t, x)) \cdot)\right. \\
& \left.\left.\quad+\operatorname{tr}\left(D^{2} f\right)(X(t, y))(\sigma(X(t, y)) \cdot, \sigma(X(t, y)) \cdot)\right)\right\} d t \\
& +\frac{2 \rho(t)}{\gamma^{2}}\|(D f)(X(t, x))(\sigma(X(t, x)))+(D f)(X(t, y))(\sigma(X(t, y)))\|^{2}|Z(t)|^{2} d t .
\end{aligned}
$$

Let us take a look at the second and third terms of (4.6). This term is not equal to 0 when $X(t, x)$ or $X(t, y)$ hits $\partial D$. By the property of $f$, these terms are negative. Taking this into account and using the Burkholder-Davis-Gundy inequality, we estimate $L^{p}$-norm of $\max _{0 \leq t \leq T^{\prime}} k(t)(0 \leq$ $\left.T^{\prime} \leq T\right)$, where $p \geq 2$. Similarly to the proof of Theorem 3.1 in [2] and Lemma 3.1 in [16], we have

$$
E\left[\max _{0 \leq t \leq T^{\prime}} k(t)^{p}\right] \leq C_{p}|x-y|^{2 p}+C_{p}^{\prime} \int_{0}^{T^{\prime}} E\left[\max _{0 \leq s \leq t} k(s)^{p}\right] d t
$$

which implies the desired result.

We prove (2). When $x \notin B\left(z, 2 \delta^{\prime} / 3\right), X^{z}(t, x, \omega)=X^{N, z}(t, x, \omega)=x$ for all $t, N$. So we assume $x \in B\left(z, 2 \delta^{\prime} / 3\right)$. If $B\left(z, \delta^{\prime}\right) \cap \partial D=\emptyset$, by the properties (i) and (ii), $X^{N, z}(t, x)$ and $X^{z}(t, x)$ never hits the boundary of $D$. Hence the classical Wong-Zakai theorem implies the assertion. Suppose $B\left(z, \delta^{\prime}\right) \cap \partial D \neq \emptyset$. Then there exists $z_{0} \in \partial D$ such that $\overline{B\left(z, \delta^{\prime}\right)} \subset B\left(z_{0}, \delta\right)$. In [2], (4.3) is proved under the conditions (A), (B) and (C) on D. By Lemma 3.1, the condition (C) holds locally in some sense. Also, $X^{N, z}(t, x), X^{z}(t, x) \in B\left(z, 2 \delta^{\prime} / 3\right)$. However, we cannot conclude that the proof in [2] works in the present case too. Because, there, first, we proved that the Euler approximation converges to the true solution in Theorem 3.1 and, second, the difference of the Euler approximation and the Wong-Zakai approximation converges to 0 in Lemma 4.6 in 2]. In the present case, the Euler approximation solution may exit from $B\left(z, 2 \delta^{\prime} / 3\right)$ and reach the boundary of $D$ outside $B\left(z_{0}, \delta\right)$ even if $x \in B\left(z, 2 \delta^{\prime} / 3\right)$. However, such a probability is small and we can prove (4.3). Let us show it more precisely. Let $X_{E}^{N, z}(t, x)$ be the Euler approximation of the solution to $(\mathrm{SDE})_{\sigma^{z}, b^{z}}$ with the starting point $x$ associated with the partition $\{k T / N\}_{k=0}^{N}$ and $\Phi_{E}^{N, z}(t, x)$ be the associated local time term. See (3.37) for the definition of the Euler approximation. Let $N$ be a sufficiently large number such that $\|b\|_{\infty} \Delta_{N}$ is small. Then by the estimate (3.2), we have

$$
\begin{aligned}
& P\left(\left\{\text { There exists a time } t \in[0, T] \text { such that } X_{E}^{N, z}(t, x) \in B\left(z, \delta^{\prime}\right)^{c}\right\}\right) \\
& \quad \leq P\left(\max _{1 \leq k \leq N}\|B\|_{\infty,[(k-1) T / N, k T / N]} \geq \varepsilon \delta^{\prime}\right) \\
& \quad \leq P\left(\|B\|_{\mathcal{H}, \theta}>\varepsilon \delta^{\prime}\left(\frac{N}{T}\right)^{\theta}\right) \leq \exp \left(-C\left(\varepsilon \delta^{\prime}\right)^{2}\left(\frac{N}{T}\right)^{2 \theta}\right)
\end{aligned}
$$


where $\varepsilon$ is a small positive number and \|\|$_{\mathcal{H}, \theta}$ denotes the Hölder norm with exponent $\theta(\theta<1 / 2)$. Thus, combining (4.8), and the moment estimates in Lemma 2.8 and Lemma 3.2 in [2] for $X, \Phi, X_{E}^{N, z}, \Phi_{E}^{N, z}$, by a similar calculation to the proof of Theorem 3.1, we obtain

$$
\begin{aligned}
& E\left[\max _{0 \leq t \leq T^{\prime}}\left|X_{E}^{N, z}(t, x)-X(t, x)\right|^{2 p}\right] \\
& \quad \leq C_{T} \Delta_{N}^{p}+e^{-C(N / T)^{2 \theta}}+C_{T} \int_{0}^{T^{\prime}} E\left[\max _{0 \leq s \leq t}\left|X_{E}^{N, z}(s, x)-X(s, x)\right|^{2 p}\right] d s
\end{aligned}
$$

which implies $E\left[\max _{0 \leq t \leq T}\left|X_{E}^{N, z}(t, x)-X(t, x)\right|^{2 p}\right] \leq C_{T} \Delta_{N}^{p}$. Similarly, the key of the proof of Lemma 4.6 in [2] is the non-positivity of the sum of second and third terms involving local times $\Phi^{N}$ and $\Phi_{E}^{N}$ in (4.49). For (SDE) $\sigma_{\sigma^{z}, b^{z}}$ too, the corresponding term involving $\Phi^{N, z}$ is non-positive. For the term $\Phi_{E}^{N, z}$, by the same reasoning as in (4.10), we have

$$
\begin{aligned}
& E\left[\int_{t_{k-1}}^{t_{k}}\left\{\rho^{N, z}(t)\left(Z^{N, z}(t), d \Phi_{E}^{N, z}(t)\right)-\frac{\rho^{N, z}(t)}{\gamma}\left|Z^{N, z}(t)\right|^{2}\left((D f)\left(X_{E}^{N, z}(t)\right), d \Phi_{E}^{N, z}(t)\right)\right\}\right] \\
& \quad \leq C_{T} e^{-C(N / T)^{2 \theta}}
\end{aligned}
$$

where $\rho^{N, z}(t)=\exp \left(-\frac{2}{\gamma}\left(f\left(X_{E}^{N, z}(t, x)\right)+f\left(X^{N, z}(t, x)\right)\right)\right)$. Consequently, in a similar way to the proof of Lemma 4.6 in [2], we obtain for any $0<\theta<1$

$$
\begin{array}{r}
\max _{0 \leq k \leq N} E\left[\left|X^{N, z}\left(t_{k}^{N}\right)-X_{E}^{N, z}\left(t_{k}^{N}\right)\right|^{2}\right] \leq C_{\theta} \cdot \Delta_{N}^{\theta / 2} \\
E\left[\max _{0 \leq t \leq T}\left|X^{N, z}(t)-X^{z}(t)\right|^{2}\right] \leq C_{T, \theta} \Delta_{N}^{\theta / 6} .
\end{array}
$$

The assertion (3) can be proved by the same argument as in the proof of Lemma 5.5 in [23].

Proof of Theorem 2.3. Let $x \in \bar{D}$ and $P_{x}$ denote the probability law of the process $X(t, x)(0 \leq$ $t \leq T)$ which exists on $C([0, T] \rightarrow \bar{D} ; w(0)=x)$. Let $c(t)(0 \leq t \leq T)$ be a point of the support of $P_{x}$ and

$$
U_{r}(c)=\left\{\omega\left|\max _{0 \leq t \leq T}\right| X(t, x, \omega)-c(t) \mid \leq r\right\} .
$$

It is sufficient to prove that for any $\varepsilon>0$ and $c$

$$
\lim _{N \rightarrow \infty} P\left(\left\{\max _{0 \leq t \leq T}\left|X(t, x)-X^{N}(t, x)\right| \geq \varepsilon\right\} \cap U_{\delta^{\prime} / 4}(c)\right)=0 .
$$

Let us define a subset of increasing numbers $\left\{s_{0}, \ldots, s_{K}\right\} \subset\left\{t_{k}^{N}\right\}_{k=0}^{N}$ so that $s_{0}=0$ and $s_{k}=$ $\max \left\{t_{l}^{N} \geq s_{k-1}\left|\max _{s_{k-1} \leq t \leq t_{l}^{N}}\right| c(t)-c\left(s_{k-1}\right) \mid \leq \delta^{\prime} / 8\right\}$. For any $c$, if $N$ is sufficiently large, then the set on the RHS in the definition of $s_{k}$ is not empty and $s_{K}=T$. Note that the set $\left\{s_{k}\right\}$ and $K$ may depend on $N$ but $\lim \sup _{N \rightarrow \infty} K<\infty$. We prove by an induction on $1 \leq k \leq K$ that for any $\varepsilon>0$

$$
\lim _{N \rightarrow \infty} P\left(\left\{\max _{0 \leq t \leq s_{k}}\left|X(t, x)-X^{N}(t, x)\right| \geq \varepsilon\right\} \cap U_{\delta^{\prime} / 4}(c)\right)=0 .
$$


First, we prove the case $k=1$. Let $s_{1}^{*}=\max \left\{t\left|\max _{0 \leq s \leq t}\right| c(s)-x \mid \leq \delta^{\prime} / 8\right\}$. Clearly, $s_{1} \leq s_{1}^{*}$ and $s_{1} \rightarrow s_{1}^{*}$ as $N \rightarrow \infty$. We prove

$$
\lim _{N \rightarrow \infty} P\left(\left\{\max _{0 \leq t \leq s_{1}^{*}}\left|X(t, x)-X^{N}(t, x)\right| \geq \varepsilon\right\} \cap U_{\delta^{\prime} / 4}(c)\right)=0 .
$$

By Lemma 4.2 (3), we have

$$
\begin{aligned}
& P\left(\left\{\max _{0 \leq t \leq s_{1}^{*}}\left|X(t, x)-X^{N}(t, x)\right| \geq \varepsilon\right\} \cap U_{\delta^{\prime} / 4}(c)\right) \\
& =P\left(\left\{\max _{0 \leq t \leq s_{1}^{*}}\left|X^{x}(t, x)-X^{N}(t, x)\right| \geq \varepsilon\right\} \cap U_{\delta^{\prime} / 4}(c)\right) \\
& =P\left(\left\{\max _{0 \leq t \leq s_{1}^{*}}\left|X^{x}(t, x)-X^{N}(t, x)\right| \geq \varepsilon\right\} \cap\left\{\max _{0 \leq t \leq s_{1}^{*}}\left|X^{x}(t, x)-X^{N, x}(t, x)\right| \geq \delta^{\prime} / 8\right\}\right. \\
& +P\left(\left\{\max _{0 \leq t \leq s_{1}^{*}}\left|X^{x}(t, x)-X^{N}(t, x)\right| \geq \varepsilon\right\} \cap\left\{\max _{0 \leq t \leq s_{1}^{*}}\left|X^{x}(t, x)-X^{N, x}(t, x)\right| \leq \delta^{\prime} / 8\right\}\right. \\
& \left.\quad \cap U_{\delta^{\prime} / 4}(c)\right) \\
& \quad P\left(\left\{\max _{0 \leq t \leq s_{1}^{*}}\left|X^{x}(t, x)-X^{N, x}(t, x)\right| \geq \delta^{\prime} / 8\right\}\right) \\
& \quad P\left(\left\{\max _{0 \leq t \leq s_{1}^{*}}\left|X^{x}(t, x)-X^{N, x}(t, x)\right| \geq \varepsilon\right\}\right) .
\end{aligned}
$$

Here we have used that for $\omega$ satisfying $\max _{0 \leq t \leq s_{1}^{*}}\left|X^{N, x}(t, x, \omega)-x\right| \leq \delta^{\prime} / 2, X^{N}(t, x, \omega)=$ $X^{N, x}(t, x, \omega)$ holds for $0 \leq t \leq s_{1}^{*}$. The estimate (4.18) and Lemma 4.2 (2) implies the case $k=1$. We prove (4.16) in the case of $k+1$ assuming the case of $k$. Let

$$
V_{\eta, k}=\left\{\omega\left|\max _{0 \leq t \leq s_{k}}\right| X(t, x)-X^{N}(t, x) \mid \leq \eta\right\}
$$

It suffices to prove

$$
\limsup _{\eta \rightarrow 0} \limsup _{N \rightarrow \infty} P\left(\left\{\max _{s_{k} \leq t \leq s_{k+1}}\left|X(t, x)-X^{N}(t, x)\right| \geq \varepsilon\right\} \cap U_{\delta^{\prime} / 4}(c) \cap V_{\eta, k}\right)=0 .
$$

Note that for $t \geq s_{k}$,

$$
X(t, x, \omega)=X\left(t-s_{k}, X\left(s_{k}, x, \omega\right), \tau_{k} \omega\right), \quad X^{N}(t, x, \omega)=X^{N}\left(t-s_{k}, X^{N}\left(s_{k}, x, \omega\right), \tau_{k} \omega\right),
$$

where $\left(\tau_{k} \omega\right)(t)=\omega\left(t+s_{k}\right)$. This identity follows from the uniqueness of strong solutions and 
$B^{N}\left(t, \tau_{k} \omega\right)=B^{N}\left(s_{k}+t, \omega\right)$ for all $k$ and $t \geq 0$. Hence,

$$
\begin{aligned}
& P\left(\left\{\max _{s_{k} \leq t \leq s_{k+1}}\left|X(t, x)-X^{N}(t, x)\right| \geq \varepsilon\right\} \cap U_{\delta^{\prime} / 4}(c) \cap V_{\eta, k}\right) \\
& \quad \leq P\left(\left\{\max _{0 \leq s \leq s_{k+1}-s_{k}}\left|X\left(s, X\left(s_{k}\right), \tau_{k} \omega\right)-X\left(s, X^{N}\left(s_{k}\right), \tau_{k} \omega\right)\right| \geq \varepsilon / 2\right\} \cap U_{\delta^{\prime} / 4}(c) \cap V_{\eta, k}\right) \\
& \quad+P\left(\left\{\max _{0 \leq s \leq s_{k+1}-s_{k}}\left|X\left(s, X^{N}\left(s_{k}\right), \tau_{k} \omega\right)-X^{N}\left(s, X^{N}\left(s_{k}\right), \tau_{k} \omega\right)\right| \geq \varepsilon / 2\right\} \cap U_{\delta^{\prime} / 4}(c) \cap V_{\eta, k}\right) \\
& \quad:=I_{1}+I_{2},
\end{aligned}
$$

where we have written $X\left(s_{k}\right)=X\left(s_{k}, x, \omega\right)$ and $X^{N}\left(s_{k}\right)=X^{N}\left(s_{k}, x, \omega\right)$ for simplicity. By Lemma 4.2 (1) and the Chebyshev inequality, we have $I_{1} \leq 4 \varepsilon^{-2} C_{2} \eta^{2}$. Let

$$
\begin{aligned}
& W_{k, \delta^{\prime}, \eta}=\left\{\max _{0 \leq s \leq s_{k+1}-s_{k}}\left|X\left(s, X\left(s_{k}\right), \tau_{k} \omega\right)-X\left(s, X^{N}\left(s_{k}\right), \tau_{k} \omega\right)\right| \leq \delta^{\prime} / 16\right\} \cap U_{\delta^{\prime} / 4}(c) \cap V_{\eta, k}, \\
& I_{3}=P\left(\left\{\max _{0 \leq s \leq s_{k+1}-s_{k}}\left|X\left(s, X^{N}\left(s_{k}\right), \tau_{k} \omega\right)-X^{N}\left(s, X^{N}\left(s_{k}\right), \tau_{k} \omega\right)\right| \geq \varepsilon / 2\right\} \cap W_{k, \delta^{\prime}, \eta}\right) .
\end{aligned}
$$

To prove $\lim \sup _{\eta \rightarrow 0} \lim \sup _{N \rightarrow \infty} I_{2}=0$, it suffices to show $\lim \sup _{N \rightarrow \infty} I_{3}=0$ for any $\eta$. We explain the reason. By Lemma 4.2 (3),

$$
\begin{aligned}
& P\left(\left\{\max _{0 \leq s \leq s_{k+1}-s_{k}}\left|X\left(s, X\left(s_{k}\right), \tau_{k} \omega\right)-X\left(s, X^{N}\left(s_{k}\right), \tau_{k} \omega\right)\right| \geq \delta^{\prime} / 16\right\} \cap U_{\delta^{\prime} / 4}(c)\right. \\
& \left.\cap\left\{\left|X\left(s_{k}\right)-X^{N}\left(s_{k}\right)\right| \leq \delta^{\prime} / 8\right\}\right) \\
& =P\left(\left\{\max _{0 \leq s \leq s_{k+1}-s_{k}}\left|X^{c\left(s_{k}\right)}\left(s, X\left(s_{k}\right), \tau_{k} \omega\right)-X^{c\left(s_{k}\right)}\left(s, X^{N}\left(s_{k}\right), \tau_{k} \omega\right)\right| \geq \delta^{\prime} / 16\right\} \cap U_{\delta^{\prime} / 4}(c)\right. \\
& \left.\cap\left\{\left|X\left(s_{k}\right)-X^{N}\left(s_{k}\right)\right| \leq \delta^{\prime} / 8\right\}\right) .
\end{aligned}
$$

By Lemma 4.2 (1), this probability goes to 0 as $N \rightarrow \infty$ by the assumption of the induction. Now we estimate $I_{3}$. For $\omega \in W_{k, \delta^{\prime}, \eta}$, we have

$$
\left|X\left(s, X^{N}\left(s_{k}, x, \omega\right), \tau_{k} \omega\right)-c\left(s+s_{k}\right)\right| \leq \frac{5 \delta^{\prime}}{16} \quad 0 \leq s \leq s_{k+1}-s_{k}
$$

and so

$$
\left|X\left(s, X^{N}\left(s_{k}, x, \omega\right), \tau_{k} \omega\right)-c\left(s_{k}\right)\right| \leq \frac{7 \delta^{\prime}}{16} \quad 0 \leq s \leq s_{k+1}-s_{k} .
$$

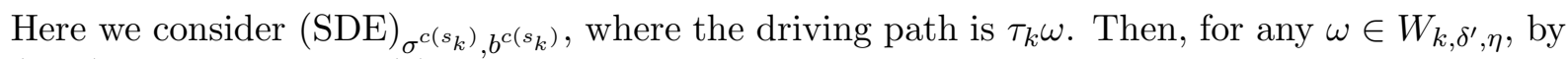
(4.25) and Lemma $4.2(3)$,

$$
X\left(s, X^{N}\left(s_{k}, x, \omega\right), \tau_{k} \omega\right)=X^{c\left(s_{k}\right)}\left(s, X^{N}\left(s_{k}, x, \omega\right), \tau_{k} \omega\right) \quad 0 \leq s \leq s_{k+1}-s_{k} .
$$

Hence, by a similar argument to the case $k=1$, we can prove $\lim \sup _{N \rightarrow \infty} I_{3}=0$ which completes the proof. 
Remark 4.3. As explained in the above proof, we estimated the difference $X^{N}-X_{E}^{N}$ in Lemma 4.6 in [2]. However, it is easy to check that we can estimate the difference $X^{N}-X$ in a similar way to the proof of $X^{N}-X_{E}^{N}$ and obtain $\max _{0 \leq t \leq T} E\left[\left|X^{N}(t)-X(t)\right|^{2}\right] \leq C_{T, \theta} \Delta_{N}^{\theta / 2}$ in the setting in [2]. In the proofs of Theorem 2.2 and Theorem 2.3 too, we can directly estimate the difference $X^{N}-X$ in the convex case and $X^{N, z}-X^{z}$ similarly. By noting this, actually, we do not need to use the Euler approximation in the above proofs too. Also, we note that Zhang [28] proved that the difference $X^{N}-X$ converges to 0 without using the Euler approximation under stronger assumptions than those in [2].

\section{References}

[1] S. Aida, Reflecting rough differential equations, arXiv:1311.6104.

[2] S. Aida and K. Sasaki, Wong-Zakai approximation of solutions to reflecting stochastic differential equations on domains in Euclidean spaces, Stochastic Process. Appl. Vol. 123 (2013), Issue 10, 3800-3827.

[3] A.M. Davie, Differential equations driven by rough paths: an approach via discrete approximations, Appl. Math. Res. Express. AMRX 2007, no. 2, Art. ID abm009, 40 pp.

[4] A. Deya, A. Neuenkirch and S. Tindel, A Milstein-type scheme without Lévy area terms for SDEs driven by fractional Brownian motion, Ann. Inst. Henri. Poincaré Probab. Stat. 48 (2012), no.2, 518-550.

[5] H. Doss and P. Priouret, Support d'un processus de réflexion, Z. Wahrsch. Verw. Gebiete 61 (1982), no. 3, 327345.

[6] P. Dupuis and H. Ishii, On Lipschitz continuity of the solution mapping to the Skorokhod problem, with applications. Stochastics Stochastics Rep. 35 (1991), no. 1, 3162.

[7] L.C. Evans and D.W. Stroock, An approximation scheme for reflected stochastic differential equations, Stochastic Process. Appl. 121 (2011), no. 7, 14641491.

[8] M. Ferrante and C. Rovira, Stochastic differential equations with non-negativity constraints driven by fractional Brownian motion, J. Evol. Equ. 13 (2013), 617-632.

[9] P. Friz and H. Oberhauser, Rough path limits of the Wong-Zakai type with a modified drift term, J. Funct. Anal. 256 (2009) no.10, 3236-3256.

[10] P. Friz and S. Riedel, Convergence rates for the full Gaussian rough paths, arXiv:1108.1099.

[11] P. Friz and N. Victoir, Multidimensional Stochastic Processes as Rough Paths Theory and Applications, Cambridge Studies in Advanced Mathematics, 120, Cambridge University Press (2010).

[12] M. Gubinelli, Controlling rough paths. J. Funct. Anal. 216 (2004), no. 1, 86-140.

[13] I. Gyöngy and P.R. Stinga, Rate of convergence of Wong-Zakai approximations for stochastic partial differential equations, Seminar on Stochastic Analysis, Random Fields and Applications VII, Progress in Probability, Vol.67, (2013), 95-130. 
[14] Y. Hu and D. Nualart, Rough path analysis via fractional calculus, Trans. Amer. Math. Soc. 361 (2009), no.5, 2689-2718.

[15] N. Ikeda and S. Watanabe, Stochastic differential equations and diffusion processes. NorthHolland Mathematical Library, 24. North-Holland Publishing Co., Amsterdam-New York; Kodansha, Ltd., Tokyo, 1981.

[16] P.L. Lions and A.S. Sznitman, Stochastic differential equations with reflecting boundary conditions, Comm. Pure Appl. Math. 37 (1984), no. 4, 511537.

[17] T. Lyons, Differential equations driven by rough signals, Rev.Mat.Iberoamer., 14 (1998), 215-310.

[18] T. Lyons and Z. Qian, System control and rough paths, (2002), Oxford Mathematical Monographs.

[19] T. Lyons, M. Caruana and T. Lévy, Differential equations driven by rough paths. Lecture Notes in Mathematics, 1908 Springer, Berlin, 2007.

[20] R. Pettersson, Wong-Zakai approximations for reflecting stochastic differential equations. Stochastic Anal.Appl. 17 (1999), no. 4, 609617.

[21] J. Ren and S. Xu, A transfer principle for multivalued stochastic differential equations. J. Funct. Anal. 256 (2009), no. 9, 27802814.

[22] J.Ren and S. Xu, Support theorem for stochastic variational inequalities. Bull. Sci. Math. 134 (2010), no. 8, 826856.

[23] Y. Saisho, Stochastic differential equations for multi-dimensional domain with reflecting boundary, Probab. Theory Related Fields 74 (1987), no. 3, 455477.

[24] L. Słomiński, On approximation of solutions of multidimensional SDEs with reflecting boundary conditions. Stochastic Process. Appl. 50 (1994), no. 2, 197219.

[25] L. Słomiński, Euler's approximations of solutions of SDEs with reflecting boundary. Stochastic Process. Appl. 94 (2001), no. 2, 317337.

[26] H. Tanaka, Stochastic differential equations with reflecting boundary condition in convex regions, Hiroshima Math. J. 9 (1979), no. 1, 163177.

[27] E. Wong and M. Zakai, On the relation between ordinary and stochastic differential equations. Internat. J. Engrg. Sci. 3 (1965) 213229.

[28] T-S. Zhang, Strong Convergence of Wong-Zakai Approximations of Reflected SDEs in A Multidimensional General Domain, DOI 10.1007/s11118-014-9394-9, Potential Analysis, 2014. 